\title{
Arbor
}

\section{Gran Bretaña ante Europa Tormenta en el Canal. El continente aislado}

\section{$M^{a}$ Dolores Elizalde}

Arbor CLXX, 669 (Septiembre 2001), 43-71 pp.

El presente artículo estudia el difícil camino de Gran Bretaña hacia la integración en la Comunidad Europea. El trabajo se inicia con un análisis de los factores que determinaron la posición británica ante Europa; explica luego el modelo de Europa deseado por los británicos; y se detiene especialmente en las distintas actitudes adoptadas por Gran Bretaña durante el proceso de integración europea, centrándose en tres ámbitos: la política, la economía y la defensa. En ese marco, el trabajo revisa las razones por las que Gran Bretaña apoyó o rechazó las distintas instituciones europeas que se fueron creando, los motivos de su alejamiento del núcleo inicial de la CEE, y las causas de su posterior incorporación a la estructura comunitaria.

\section{Europa desde la perspectiva británica}

Durante el período que nos ocupa, es decir, desde la Primera Guerra Mundial, en que comenzó a hablarse de la construcción de una Europa unida, a los primeros años de la década de 1970, en que Gran Bretaña se incorporó efectivamente a la Comunidad, la nación británica fue uno de los pilares europeos, una de sus grandes potencias, y su influencia sobre el conjunto necesariamente hubo de ser significativa. No obstante, 
dentro de ese conjunto, Gran Bretaña ocupó una posición especial. La expresión Tormenta en el Canal, el Continente aislado no deja de ser una broma, pero tiene un fondo de realidad. Quizás debido a su insularidad, quizás por su extraversión ultramarina, quizás por la importancia de su Imperio, Gran Bretaña no sólo se sintió integrada en el continente europeo. Evidentemente, era Europa pero, al tiempo, era más que Europa, algo más allá de Europa.

\section{Elementos que condicionaron la visión británica de Europa}

Existieron varios factores que incidieron en la actitud británica ante Europa. En primer lugar, Gran Bretaña era una democracia asentada, con un sistema parlamentario sólido, un modelo político consolidado y una economía desarrollada. No necesitaba apoyos externos para asegurar su régimen interior. Tampoco para impulsar la democratización de sus instituciones o la modernización de sus estructuras productivas. No precisaba, pues, que Europa amparara su evolución ${ }^{1}$. En segundo lugar, Gran Bretaña no sólo era una potencia europea, sino que se proyectaba y prolongaba a través de un Imperio extendido por todo el mundo. Los intereses de esa construcción imperial primaban sobre cualquier otra consideración y determinaban su política exterior ${ }^{2}$. Y tercero, desde fines del XIX, Gran Bretaña había desarrollado una estrecha relación con Estados Unidos, la special relationship, que condicionaba sus relaciones con las demás potencias y la dotaba de una dimensión atlántica especialmente acusada dentro del conjunto europeo.

Desde una posición definida por la encrucijada de esos factores, la política que tradicionalmente había desarrollado Gran Bretaña respecto a Europa se había caracterizado por tratar de mantener el Continente neutralizado y por intentar preservar el equilibrio entre las distintas alianzas, sin que ninguna potencia o grupo de potencias destacaran por encima de las demás, y así poder dedicarse a sus verdaderos intereses internacionales, que eran fundamentalmente extraeuropeos. En principio, Gran Bretaña no era favorable a pactos que limitaran su libertad, o que restaran un ápice a su soberanía. Pero tampoco quería ser eternamente el vigía de Europa, siempre buscando el equilibrio entre fuerzas opuestas, siempre conteniendo posibles conflictos ${ }^{3}$. La aspiración británica era mantener la paz y la estabilidad en el Continente. No deseaba una Francia excesivamente fuerte, pero tampoco quería una Alemania humillada e incapaz de reconstruirse, y recelaba de la Rusia soviética. Defendía la existencia de unos valores europeos comunes que estaba dispuesta a defender frente a cualquier agresión. 
Gran Bretaña ante Europa.....

Pretendía que tales principios fueran respetados por todas las naciones europeas y que no hubiera excesivas rivalidades, ni invasiones territoriales. Para preservar ese orden, desde la perspectiva británica, lo óptimo sería crear unas instituciones internacionales intergubernamentales y no transnacionales, que garantizaran la estabilidad y el desarrollo político y económico alcanzado. Por ello, tras la Primera Guerra Mundial, y aún más después de la Segunda, apoyó la formalización de un acuerdo colectivo y la articulación de unos organismos comunes que velaran por Europa ${ }^{4}$. Aún sin ser entusiasta de dichos mecanismos, los consideró el mal menor. Sus reticencias ante esos métodos se reflejaron en su particular y errático camino hacia la integración europea. En su peculiar visión de Europa hubo varios problemas que preocuparon especialmente a los británicos.

\section{El papel internacional de una Europa unida desde la perspectiva británica}

Uno de los objetivos británicos en el proceso de la formación de una Europa unida fue procurar que no desembocara en la construcción de un regionalismo europeo que obstaculizara la ejecución de una política internacional concebida en términos globales, que era la perspectiva desde la cual Gran Bretaña se planteaba su acción exterior ${ }^{5}$. A pesar de que había perdido el liderazgo militar y económico que había ejercido en el siglo XIX, Gran Bretaña seguía siendo una potencia con intereses en todo el mundo y defendía el mantenimiento de la libertad de comercio en las transacciones internacionales. Su industria continuaba siendo lo suficientemente poderosa como para competir ventajosamente en un régimen de puertas abiertas. Tenía especial interés en mantener esa inclinación globalista como rasgo esencial de su política exterior y pretendía que el proceso europeísta no impidiera ni perjudicara tal sesgo. Aunque deseaba que Europa pudiera convertirse en un tercer bloque con peso específico en la escena internacional, no quería que ello limitara el radio de su proyección exterior. Ya Winston Churchill había defendido que Gran Bretaña debía ser el eje de tres esferas de influencia interconectadas: el Imperio británico, el mundo atlántico, y Europa. Esta doctrina, con ligeras variaciones dependiendo del énfasis que se diera a cada uno de esos tres elementos, fue aceptada por los sucesivos ministros de exteriores británicos de la postguerra, el laborista Ernest Bevin y el conservador Anthony Eden ${ }^{6}$. Esa triple orientación exterior, junto al peso de su pasado imperial, hicieron que Gran Bretaña fuera una potencia con una perspectiva globalista y con preocupaciones 
internacionales más amplias que las de otras naciones europeas. A ello contribuían, además, varias circunstancias: no había sufrido unos efectos internos tan devastadores como otros países continentales, no tenía la misma conciencia de debilidad nacional, ni sentía igual necesidad de garantizar sus fronteras y su seguridad territorial7. Su

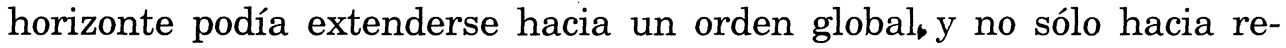
querimientos nacionales o continentales.

\section{El modelo de Europa: ¿federal o intergubernamental? ¿una unión política, económica, militar...?}

La integración europea es un término que ha estado abierto a diferentes interpretaciones. En el tiempo que nos ocupa, los primeros pasos de la construcción de una Europa unida, para la élite gobernante británica significaba ante todo un entendimiento económico que permitiera incentivar la reconstrucción de las economías nacionales y definiera un área de libre comercio en Europa, el cual -a su vezse adaptara a un mundo de puertas abiertas. Dicha élite era más reticente a la integración política y militar, campos en los cuales prefería hablar de cooperación entre gobiernos soberanos. Defendía que los acuerdos debían establecerse entre gobiernos independientes que desearan aunar sus respectivas políticas exteriores e integrar sus objetivos internacionales para tener más peso en la escena mun$\mathrm{dial}^{8}$. La perspectiva británica contrastaba con la de los seis miembros fundadores de la CEE, que adoptaron una política más intervencionista. En el terreno económico, en vez de hablar de libertad de comercio, estos países se centraron en lograr acuerdos consensuados y en unificar sus respectivas políticas. Para ellos, además, la integración económica no era un fin por si misma, sino un paso previo hacia el verdadero objetivo: la unión política de Europa, proceso en el cual apoyaron la creación de unas instituciones europeas comunes que tuvieran amplias atribuciones.

En la conciencia nacional británica existían una serie de ideas y sentimientos muy arraigados que dificultaron su integración en unas instituciones europeas organizadas según los criterios de los demás países. En primer lugar, el temor a perder soberanía nacional y capacidad de decisión y de acción en el seno de una institución supranacional ${ }^{9}$. Segundo, el deseo de mantener su independencia económica y defender el libre comercio. Tercero, unas claras reticencias ante las limitaciones que conllevaría una política defensa consensuada entre 
Gran Bretaña ante Europa.....

los países europeos. Cuarto, un fuerte rechazo ante la imposición de una burocracia europea ${ }^{10}$ y de unas leyes comunes que recortaran las competencias del Parlamento británico ${ }^{11}$. La suma de esas consideraciones pesaron en la actitud británica a la hora de construir una Europa unida, e hicieron que Gran Bretaña se inclinara por un modelo que no restringiera su libertad ni recortara su soberanía nacional ${ }^{12}$.

\section{La opinión pública británica ante Europa}

La opinión pública británica sólo de manera gradual, y aún así sin mucho entusiasmo, fue apoyando la integración británica en Europa. Mientras que en otros países europeos después de la Segunda Guerra Mundial había surgido un cierto rechazo hacia el fervor nacionalista que podía amenazar la paz colectiva, y se presentaba la integración europea como un proceso deseable para evitar riesgos futuros de guerra, en Gran Bretaña la contienda del 45 había servido para reforzar el orgullo de la identidad nacional y para afirmar su condición de británicos. Por ello, no existía un substrato ideológico que impulsara a la unidad europea: ésta era presentada en términos puramente pragmáticos, como algo que sería positivo para la economía nacional y para reforzar la posición internacional de Gran Bretaña ${ }^{13}$.

Para los británicos la integración europea se planteaba como un asunto de conveniencia, y no como la realización de un ideal ${ }^{14}$. La discusión se centraba sobre si los costes de la incorporación eran mayores que los beneficios, o si ocurría lo contrario, y esas valoraciones prácticas eran las que decidían las posiciones de partidarios y detractores. Pocas veces se habló de cumplir un destino, de alcanzar un anhelo político, ni se señaló que -a pesar de los costes que la integración pudiera tener para Gran Bretaña- se estaba sirviendo a un bien superior, a elevados propósitos colectivos, comunes a toda Europa. La retórica estuvo más ausente en el discurso británico que en el de otros países. Esa diferencia de actitudes entre pragmáticos e idealistas se evidenció aún más ante el hecho de que Gran Bretaña no se integró efectivamente en la Comunidad Europa hasta que el crecimiento económico de los sesenta no se hubo acabado. Su incorporación efectiva a los organismos europeos coincidió con una persistente recesión de su economía, en la cual los británicos sintieron de forma más acuciante la necesidad de integrarse en Europa ${ }^{15}$.

También la posición geográfica favoreció la diferenciación. Para la Europa continental la integración y el intercambio eran realidades 
más palpables y cotidianas: las gentes de diferentes nacionalidades tenían un contacto mucho más estrecho y cruzaban fronteras con más frecuencia. Por contra, los británicos siguieron considerando al resto de los europeos como overseas hasta fines de los años ochenta. Una realidad diferente, ajena a ellos mismos.

\section{Los partidos políticos y Europa}

La posición respecto a Europa fue uno de los asuntos más constantemente debatidos en el seno de la política interior británica. Ninguno de los partidos mayoritarios tuvo una postura clara e inequívoca en favor de una plena integración en Europa. Siempre señalaron determinados matices que condicionaban esa integración. Los pequeños partidos británicos de centro, -los liberales y los socialdemócratas-, fueron mucho más entusiastas en su apoyo a la incorporación a Europa. Por contra, los conservadores y los laboristas estuvieron divididos en sus opiniones, y sus líderes se vieron forzados a adoptar una línea media entre las distintas tendencias existentes en sus respectivas formaciones.

En el Partido Conservador se podían distinguir tres tendencias: los tories más tradicionales que eran los más firmes oponentes a una participación en la Comunidad; el ala liberal, que apoyaba la integración siempre que se respetaran los principios de libre mercado; y los pragmáticos - como Edward Heath- que eran los más claros defensores de la adherencia a Europa, dispuestos a aceptar incluso directrices económicas desde Bruselas siempre que contribuyeran a mejorar la economía británica ${ }^{16}$.

También entre los laboristas se podían señalarse tres fracciones: primero, los modernizadores, que no se diferenciaban demasiado en sus motivaciones de sus homólogos conservadores -Wilson-; segundo, los tradicionalistas, a los que disgustaba cualquier tipo de integración en Europa: eran nacionalistas que pensaban que la Commonwealth era la única organización internacional en la cual Gran Bretaña debía cooperar económicamente -Hugh Gaitskell-; y tercero, la izquierda del partido que veía a la Comunidad europea como una organización capitalista a la que por tanto rechazaban ${ }^{17}$.

Veamos ahora cómo la concepción de Europa y la idea de la integración de Gran Bretaña en una Europa unida se fue transformando a lo largo del siglo, dependiendo de la evolución interior, de la situación económica general, del contexto internacional y de la posición exterior desempeñada por Gran Bretaña. 


\section{Del entusiasmo europeísta tras la Segunda Guerra Mundial al período errático de los cincuenta}

Cuando Gran Bretaña se integró en la Comunidad Económica Europea, en 1973, venía de un período errático, en el cual se habían entablado continuas discusiones en favor y en contra de la incorporación británica a la CEE, y se habían producido sucesivos avances y retrocesos. En los últimos veinte años se habían sucedido diferentes situaciones: en los cuarenta, el período de mayor entusiasmo europeísta; en los cincuenta, un tiempo de apoyo británico a las iniciativas europeístas, que a la postre resultó fallido en gran medida; en los sesenta, el vano intento de MacMillan y de Wilson para integrarse en la estructura europea; y en los setenta, el definitivo impulso dado por Edward Heath para que Gran Bretaña se convirtiera en miembro de pleno derecho de la Comunidad Europea.

Tras la Segunda Guerra Mundial comenzó una etapa de entusiasmo europeísta. La confrontación se vivió, no como un enfrentamiento territorial, sino como una guerra ideológica, en la cual los países aliados habían luchado en defensa de una serie de principios que caracterizan la esencia de Europa, definida -entre otros rasgos- por la democracia, el parlamentarismo, el respeto a la libertad y la defensa de los derechos individuales. Después de la contienda se inició un fuerte movimiento en favor de unos acuerdos europeos que ayudaran a preservar esos valores y que impidieran otra guerra continental originada por rivalidades nacionales. Ese ambiente se reflejó a través de sucesivas reuniones internacionales, y se concretó de forma inmediata en la creación de instituciones europeas plasmadas en el Tratado de Bruselas, el Congreso de La Haya, la ratificación de la OTAN, o la configuración del Consejo de Europa.

En ese contexto, Gran Bretaña se debatió entre el entusiasmo por el discurso europeísta y los recelos ante una posible pérdida de soberanía. Como ya hemos visto, se mostró reticente a las organizaciones supranacionales que pudieran cuestionar las atribuciones nacionales. Manifestó su disposición a la colaboración de las economías nacionales y su apoyo a los pactos internacionales encaminados a resolver conjuntamente los problemas planteados en el Continente. Pero expresó también sus reservas ante una integración política que fuera más allá de un compromiso puntual para cada caso concreto. Los británicos eran firmes partidarios de que cualquier tipo de acuerdo se adoptara desde posiciones gubernamentales.

Sin embargo, en el Continente se barajaban distintas ideas respecto a lo que debía ser una Europa unida. Nada más acabar la Segunda 
Guerra Mundial surgieron propuestas para reorganizar Europa sobre una base federal. El movimiento llegó a concretarse, en Diciembre de 1946, en la formación de la Unión Europea de Federalistas (UEF) cuyo propósito era crear unos Estados Federales de Europa. La rápida reconstrucción de los estados nacionales tras la guerra impidió esas aspiraciones. En la Conferencia de La Haya de 1948 ya no se discutió sobre las bases de un modelo federalista europeo. En cualquier caso, tal alternativa no fue aceptada por el Gobierno británico. Clement Attlee declaró desde el primer momento que no apoyaría la creación de una Europa federal. Gran Bretaña era claramente partidaria de respetar los sistemas políticos nacionales ${ }^{18}$.

Frente a las propuestas iniciales de federalismo, triunfaron las tesis de una progresiva integración europea por sectores. A pesar de que el Gobierno británico aparentemente era favorable a ese tipo de integración, se resistió a incorporarse en las principales instituciones europeas creadas en la época. Rechazó formar parte de la Comunidad Europea del Carbón y del Acero y de la Comunidad Europea de Defensa, y no corraboró los pactos adoptados en la Conferencia de Messina ni en los Tratados de Roma. Sólo aceptó participar en aquellas instituciones de carácter intergubernamental en las que no tuviera que renunciar a su independencia ni a su capacidad de decisión. De esa manera, Gran Bretaña se automarginó del inicial núcleo fuerte europeo. Fue una gran oportunidad perdida que a menudo se ha enjuiciado como una notable falta de juicio político respecto a lo que dichas organizaciones iban a suponer en el futuro de Europa ${ }^{19}$.

En los primeros tiempos, en la política británica primaron todavía los recelos hacia una organización supranacional que pudiera limitar su soberanía, y ello le hizo iniciar un período errático, que se extendió desde fines de los años cuarenta hasta fines de la década de los cincuenta, en el cual, aunque consideraba deseable la existencia de unas estructuras europeas poderosas, se resistió a alinearse en ellas bajo parámetros que no respetaran su independencia gubernamental.

\section{Gran Bretaña ante la integración política de Europa}

En una reunión celebrada el 13 de Agosto de 1945 en el Foreign Office, Ernest Bevin, recientemente nombrado ministro de exteriores en el gobierno laborista de Clement Attlee, declaró que el principal objetivo de su política europea era establecer estrechas relaciones con los países occidentales, meridionales y septentrionales del Continente en el campo político, económico y comercial. El primer paso para ello 
debía ser resolver sus problemas con Francia con el fin de consolidar un núcleo sólido en torno al cual se cohesionaran posteriormente las demás naciones. Proponía posponer la construcción de un grupo occidental europeo más amplio hasta la consecución de ese primer objetivo y hasta que tuvieran mejor estudiadas las posibles reacciones soviéticas ante tal circunstancia ${ }^{20}$.

La idea de un grupo de potencias europeas occidentales liderado por Gran Bretaña había sido sugerido por el Foreign Office ya en 1944 con miras estratégicas y políticas, pero había encontrado la oposición del Primer Ministro, Winston Churchill, que argumentó que formar una alianza con países tan débiles sería una fuente de problemas para Gran Bretaña. No excluía la posibilidad de que en el futuro se trabajara sobre esa idea, pero previamente se debía conseguir una alianza con Francia ${ }^{21}$. Bevin retomó ese planteamiento de Churchill $\mathrm{y}$, antes de intentar formar un grupo europeo, quiso concertar un acuerdo con los franceses. Sin embargo, su noción de la cooperación europea era más amplia que la del dirigente conservador. Junto a planteamientos políticos y defensivos, Bevin incidía también en la colaboración económica, lo cual era lógico dada su formación como líder sindical. No obstante, en los dieciocho meses posteriores al fin de la contienda no se hizo nada para formar un grupo europeo. En primer lugar, no fue fácil conseguir un acuerdo con los franceses debido a las exigencias que éstos planteaban en sus fronteras del Este y en la zona del Ruhr. Por otro lado, los británicos temían las reacciones de los soviéticos, que veían con recelo la posible formación de un núcleo occidental unido, y eso les hacía avanzar con pies de plomo. Finalmente, en la primavera de 1947, las circunstancias empezaron a cambiar: el 4 de Marzo se firmó un tratado de alianza entre Francia y Gran Bretaña; además, las relaciones con Moscú parecieron relajarse tras la enunciación de la Doctrina Truman en ese mismo mes de Marzo. Bevin se sintió libre al fin para promover la creación de un grupo europeo occidental que compartiera sus planteamientos políticos, económicos y defensivos. Para Gran Bretaña comenzaba entonces el camino hacia la construcción de un nuevo concepto de Europa.

El primer paso para conseguir ese objetivo se dio en el terreno estratégico. En Mayo de 1947, Sir Orme Sargent, Permanent Head of the Foreign Office, solicitó a los jefes de su staff su opinión sobre las ventajas que tendría la conclusión de tratados de alianza con Bélgica y Holanda, que siguieran el modelo ya experimentado con Francia -acuerdos explícitamente dirigidos a evitar una agresión alemana- ${ }^{22}$. Los miembros de su equipo señalaron que aunque nominalmente esas 
alianzas estuvieran dirigidas sólo a evitar una ofensiva alemana, en la práctica debían servir para fortalecer la posición estratégica de Gran Bretaña de una manera más general: lo realmente deseable sería lograr una asociación capaz de contrarrestar cualquier acción ofensiva contra Europa occidental. Por ello, recomendaban la firma de acuerdos militares con los demás países del Oeste europeo ${ }^{23}$.

En el momento que parecía estar formándose un primer embrión de una alianza europea, -todavía sólo defensiva, pero que progresivamente apuntaba a extenderse a otros campos-, la situación se vio transformada por un discurso que el general Marshall pronunció en Harvard el 5 de junio de 1947. En él explicaba la creación de un plan norteamericano que pretendía contribuir a la reconstrucción económica de Europa. Ello hizo que la cooperación europea se trasladara, de los planteamientos militares y estratégicos en que se estaba moviendo, al campo económico.

Ese planteamiento tampoco era ajeno a las intenciones británicas. Ya en Enero de 1947, Bevin había planteado al Foreign Office la conveniencia de fomentar la unidad económica europea. Temía que la Unión Soviética intentara extender su influencia no sólo entre los países del Este de Europa, sino también sobre el Oeste, y consideraba que para prevenir algo similar lo mejor sería reforzar los lazos políticos y económicos que unían a las naciones occidentales ${ }^{24}$. Sin embargo, los responsables del área económica dentro del Gobierno británico no estaban tan convencidos de las ventajas de establecer unos compromisos económicos con los demás países europeos que les convirtiera en un conjunto unificado. Sir Stafford Cripps, President of the Board of Trade, y Hugh Dalton, Chancellor of the Exchequer, señalaron que tal actitud podría perjudicar los intereses del Imperio, dificultar sus relaciones con sus socios norteamericanos, y distorsionar el orden económico internacional, por lo que se mostraron reacios a establecer una política aduanera europea común. Por ello el asunto quedó de momento paralizado $^{25}$. Cuando, durante las discusiones del Plan Marshall, se hizo evidente que los Estados Unidos no sólo no se oponían a una unión económica europea -incluida la aduanera-, sino que incluso eran favorables a esa idea, Bevin convenció a su Gabinete para que examinara de nuevo la cuestión, sugiriendo que en esa unión aduanera podría tener cabida la Commonwealth.

Sin embargo, el plan Marshall no desvió del todo la atención hacia el campo económico, y pronto el proyecto europeísta se amplió con nuevos contenidos, esta vez de carácter internacional. En el Otoño del 47, Bevin comenzó a trabajar sobre la idea de una alianza europea 
Gran Bretaña ante Europa.....

que pudiera convertir a Europa Occidental en un tercer bloque situado entre Estados Unidos y la Unión Soviética. El Gobierno británico manifestó que, puesto que la división de Europa parecía inevitable, dada la actitud rusa, consideraba necesario organizar a los países occidentales como una unidad fuerte y coherente en el escenario internacional. La ayuda norteamericana ofrecía una oportunidad para iniciar un camino que condujera a tal objetivo, pero las naciones europeas debían incrementar sus políticas de colaboración. La intención británica era implicar en ese proyecto a Gran Bretaña, Francia, Bélgica, Holanda, Portugal, Italia e Irlanda ${ }^{26}$. Tal fue la génesis de la propuesta presentada por Bevin para crear una unión occidental, presentada en la Cámara de los Comunes el 22 de Enero de 1948, y reflejada en el Tratado de Bruselas de 17 de Marzo de 1948. La iniciativa británica contemplaba la cooperación política, económica, cultural, social y militar. Según planteamientos explícitos pretendía promover una completa reorganización de la Europa occidental, y no solo en el plano económico o en el defensivo ${ }^{27}$. A pesar de las buenas intenciones mostradas en esa propuesta, y del eco que ésta encontró en el congreso de Bruselas, Bevin se encontró con dos iniciativas que torpedearon sus planes para establecer una unión occidental bajo premisas británicas.

La primera fue la creación de la Organización Europea de Cooperación Económica (OECE), un organismo diseñado para administrar la ayuda del Plan Marshall. Se acordó que fuera en el seno de esta institución donde se decidieran las cuestiones económicas que afectaran a los países occidentales. La OECE se regiría por acuerdos y cooperación intergubernamental. La orientación de esta organización encajaba en los planteamientos británicos, pero se limitaba a temas estrictamente económicos, y no era tan amplia como la unión europea propuesta por Bevin en Bruselas. La segunda circunstancia fue el debate respecto al carácter que debían tener las instituciones europeas, cuestión suscitada en el Congreso de la Haya, celebrado en Mayo de 1948. Churchill, entonces líder de la oposición, fue invitado a presidir la reunión, lo cual fue mal acogido por los laboristas en el poder que desde el principio se sintieron inclinados a boicotear los resultados de ese congreso. Pero es que, además, en La Haya se discutió nuevamente en torno a la conveniencia de adoptar - para el proceso de cooperación europea- planteamientos federalistas o proseguir con las premisas intergubernamentales adoptadas en Bruselas. Al Gobierno británico no le gustó nada el proyecto de crear un Parlamento europeo con amplias atribuciones propuesta por los franceses, por lo que inicialmente declinó formar parte del mismo ${ }^{28}$. Sin embargo, los Estados Unidos apoyaron la ini- 
ciativa francesa, y Bevin comprendió que sería poco prudente enquistarse en una actitud negativa que tuviera consecuencias perniciosas para todo el conjunto europeo. Así que tomó la decisión de respaldar tal propuesta pero fijando unos límites. El Parlamento no debía organizarse sobre bases federalistas, sino que debía convertirse una especie de consejo de ministros europeo, de caracter consultivo, integrado por delegaciones gubernamentales que se reunieran simplemente dos veces al año, y que tuviera un secretariado permanente ${ }^{29}$. A fines de Noviembre de 1948, se reunió en París una comisión con objeto de discernir qué era más conveniente entre las dos propuestas presentadas: una asamblea parlamentaria ejecutiva o un comité de representantes gubernamentales consultivo.

La delegación británica en París estuvo presidida por Hugh Dalton. Las instrucciones que recibió de Bevin fueron trabajar para construir una institución en la que estuvieran representadas las cinco potencias signatarias del Tratado de Bruselas, que eventualmente podrían ampliarse a los dieciséis miembros de la OECE. Su cometido sería coordinar a los distintos países en temas económicos, defensivos o cualquier otro problema común que pudiera plantearse en Europa. Lo cual se haría desde un planteamiento de cooperación gubernamental y no desde una asamblea que pudiera tomar decisiones independientes que luego chocaran con las posiciones de los distintos gobiernos ${ }^{30}$. Finalmente se llegó a un compromiso: en Mayo de 1949 se aprobó la creación del Consejo de Europa compuesto por dos instituciones: un Consejo de Ministros y una Asamblea Parlamentaria consultiva. El Gobierno británico confiaba en que esa fórmula funcionara correctamente, mediante un complejo mecanismo: un trabajo previo de la asamblea en torno a las cuestiones en debate, cuyas consideraciones fueran presentadas al Consejo de Ministros que, tras la consulta con sus respectivos gobiernos, decidiera los asuntos que podían ser aprobados conjuntamente. Sus esperanzas pronto se vieron defraudadas ante la dificultad de tal funcionamiento, por lo que las disensiones británicas referentes a los procedimientos a seguir en el Consejo de Europa fueron constantes.

De esta forma, las propuestas de los laboristas británicos respecto a la construcción política europea tuvieron un difícil engarce con los planteamientos promovidos por otros países. A pesar de que los ingleses querían una Europa occidental fuerte y unida, rechazaron tajantemente cualquier planteamiento supranacional. La posibilidad de que Gran Bretaña renunciara a una parte de su soberanía en bien de la comunidad resultaba puro anatema para ellos. Ese fue uno de los desacuerdos básicos entre británicos y franceses respecto al futuro de Europa. Mien- 
Gran Bretaña ante Europa.....

tras que éstos últimos optaron por fórmulas supranacionales, los laboristas británicos se opusieron frontalmente a ellas. Cuando los conservadores volvieron al poder, en Octubre de 1951, se esperaba de ellos un planteamiento más favorable al desarrollo político europeo desde bases integradoras. Sin embargo, ya en Noviembre de 1951, Sir David Maxwell Fyfe, ministro del Interior y destacado pro-europeísta, dejó sentado que su partido tampoco apoyaría la propuesta de que el Consejo de Europa se convirtiera en una especie de gobierno europeo supranacional.

\section{Gran Bretaña ante la integración económica europea}

Como una vía alternativa en el proceso de integración, que no produjera tantas reticencias como la unión política, se promovió la progresiva confluencia económica. Sin embargo, pronto se demostró que este camino tampoco conseguía los resultados apetecidos ni lograba aunar a todos los países en un proyecto común.

La Administración británica se dividió respecto a la conveniencia de apoyar una unión económica europea. Inicialmente, el Treasury y el Board of Trade se opusieron a ella, argumentando que influiría negativamente sobre los compromisos establecidos con la Commonwealth; señalaron también que los Estados Unidos se podrían sentir ofendidos si se anteponía la pertenencia a un grupo regional a la defensa común que hasta entonces habían realizado los dos países conjuntamente en favor de un orden económico mundial de libre comercio $^{31}$. El Foreign Office, por el contrario, se mostró favorable a la idea de una unión económica que pudiera facilitar la existencia de una Europa unida en otras cuestiones. El servicio exterior británico era partidario de crear un bloque europeo fuerte que pudiera dialogar con Estados Unidos y con la Rusia Soviética en términos de mayor igualdad de lo que era capaz de hacer Gran Bretaña sola. Además, este departamento consideraba que la integración en un grupo económico europeo poderoso contrarrestaría la extensión del comunismo en Europa ${ }^{32}$.

Como se ha señalado anteriormente, un paso fundamental en la integración económica europea fue la creación de la Organización para la Cooperación Económica Europea (OECE) en Abril de 1948. En origen fue una agencia para la distribución del dinero que Estados Unidos aportaba para la reconstrucción europea a través del Plan Marshall. Se ideó para promover la unidad de una Europa Occidental próspera y estable. Pero no respondió fielmente a esos objetivos, sino que se dedicó fundamentalmente a reducir las restricciones en el comercio entre los estados 


\section{$M^{a}$ Dolores Elizalde}

miembros y a dirigir un crecimiento económico común. En 1961 fue reemplazada por la Organización de Cooperación y Desarrollo Económico (OCDE), que incluía además a Estados Unidos y Canadá. Gran Bretaña apoyó inicialmente los fines de esta institución, pero pronto empezó a mostrar reticencias ante su labor. $\mathrm{Al}$ año de entrar en funcionamiento, el Gobierno británico señaló que los objetivos que justificaban este proyecto se había perdido en gran medida, y que aunque estaban dispuestos a hacer sacrificios temporales en sus modos de vida y a correr algunos riesgos en beneficio de la reconstrucción europea, el Ejecutivo no podía permitir que se dañara irreversiblemente la estructura económica de su nación ${ }^{33}$. Meses más tarde, en Octubre de 1949, Bevin era aún más explícito en su falta de compromiso con la OECE, indicando que el Gobierno de su Majestad no secundaría ningún proyecto que significara perder libertad de acción en su propia política presupuestaria y creditícea, así como en el manejo de sus reservas, ni tampoco que amenazara el equilibrio entre el dolar y la libra esterlina, o que perjudicara la política preferencial desarrollada hacia su Imperio ${ }^{34}$. Finalmente, en Noviembre de 1949, el Gobierno manifestó que Gran Bretaña no integraría su economía en un plan europeo que pudiera distorsionar sus compromisos o perjudicar los intereses de la Commonwealth y del resto del Sterling Area ${ }^{35}$.

Desde esa posición de escepticismo respecto a la integración económica europea en la que ya se encontraba, el Gobierno británico tuvo que enfrentarse con una proposición que aún le pareció más alarmante. En Mayo de 1950 se presentaba a la prensa el Plan Schuman que proponía la creación de una Comunidad Europea del Carbón y del Acero (CECA). Ello significaba que se elegían dos sectores para iniciar la integración económica europea: el carbón y el acero, industrias ambas con problemas que podían resolverse mejor en común que a nivel nacional, regulando la producción y equilibrando la distribución entre países de forma consensuada entre todos ellos. La idea de una autoridad central que organizara la obtención y reparto del carbón y del acero fue propuesta por Robert Schuman. El Gobierno británico fue invitado a participar en esa iniciativa, pero rechazó hacerlo ante la exigencia del Gobierno francés de que todas las naciones integrantes en el pacto aceptaran por adelantado los principios del supranacionalismo ${ }^{36}$.

En las discusiones previas a la decisión sobre la conveniencia de que Gran Bretaña se incorporara a esta institución, de nuevo se produjo una divergencia entre los ministerios de carácter económico y el servicio exterior. Los responsables de la economía consideraron que la pertenencia a la CECA perjudicaría a la industria británica del acero y limitaría sus posibilidades de relación a nivel mundial. El Foreign Office, por contra, se pronunció en favor de una iniciativa que contemplaba la 
Gran Bretaña ante Europa.....

reincorporación de Alemania a una organización que englobaba a toda la Europa occidental, lo cual consideraba altamente positivo para el desarrollo de la política internacional. Además, temía que en el caso de que Gran Bretaña no se integrara en la CECA, se quedara fuera de un tercer bloque mundial. Por ello los responsables del sector exterior sugirieron que Gran Bretaña debía aceptar algún sacrificio económico en aras de una posición internacional más fuerte y de no quedar aislada.

Ante la diferencia de opiniones, el Gobierno laborista decidió rechazar la incorporación a la CECA. Bevin declaró que el Plan Schuman recuperaba planteamientos federalistas, contrarios al método de integración gradual y sectorial defendido por Gran Bretaña. Stafford Cripps criticó el Plan por discrepar de la idea de la comunidad atlántica y tender hacia una federación europea. A los británicos les había molestado también que Schuman presentase la propuesta sin haberles consultado previamente su opinión, máxime cuando tuvieron constancia de que había hablado de sus intenciones con los norteamericanos. En cualquier caso, el Gobierno británico no rechazó el Plan Schuman de forma inmediata, sino que creó un grupo de expertos con el fin de que estudiaran las implicaciones de la incorporación británica, aunque el estudio resultante no fue tomado en excesiva consideración ${ }^{37}$.

Si bien en otras ocasiones el Gobierno francés había apoyado a Gran Bretaña, en el caso de la CECA, no le dio muchas alternativas. A pesar de conocer bien la oposición británica al supranacionalismo y su apuesta por la cooperación intergubernamental, los franceses exigieron que todas las partes aceptaran la primera fórmula como paso previo a las discusiones sobre el Plan Schuman. La respuesta británica no se hizo esperar: contestó diciendo que no podía participar en los debates desde las bases propuestas ${ }^{38}$. Aún así, el Gobierno continuó discutiendo sobre la conveniencia de integrarse en el proyecto, para lo cual creó una comisión ministerial y otra de funcionarios que estudiaran las ventajas y desventajas de la propuesta y las condiciones en las cuales se debería producir la integración británica. La cuestión quedó, pues, abierta para el Gobierno británico. Sin embargo, nunca pareció que hubiera llegado el momento adecuado para integrarse en esta institución y finalmente Gran Bretaña quedó al margen de ella.

\section{Gran Bretaña ante la integración militar europea}

En principio se había pensado que el campo militar sería el último sector que se integraría por las reticencias que todos los países mostraban ante la posibilidad de tener que aceptar restricciones en sus 
respectivas políticas de defensa nacional. Sin embargo, la guerra de Corea de 1950 aceleró el planteamiento del tema. Corea tenía una división territorial similar a la de Alemania, con una zona meridional bajo la influencia capitalista, y otra septentrional bajo la esfera comunista. Cuando Corea del Norte invadió el Sur, los Estados Unidos se apresuraron a acudir en ayuda de ésta última, sugiriendo que los países europeos realizaran un esfuerzo similar, con el fin de frenar la expansión de la Unión Soviética. Ello implicaba rearmar a Alemania para que pudiera defender sus fronteras orientales. Tal posibilidad despertó la alarma de Francia, por lo que rápidamente su Gobierno presentó una contrapropuesta plasmada en el Plan Pleven -en nombre del primer ministro francés, que fue quien lo propuso en Febrero de 1951-. Su objetivo era lograr una política defensiva común para Europa occidental, en vez de impulsar respuestas militares nacionales independientes que implicarían aceptar la militarización unilateral alemana.

En las conversaciones intergubernamentales entabladas con objeto de crear esa estructura militar europea se propuso que dicha fuerza multinacional se integrara en una institución política europea y se constituyera un ministerio europeo de defensa. La propuesta fue aceptada por la mayoría de los gobiernos y, en Mayo de 1952, se presentó un primer documento en el que se esbozaba la formación de una $E u$ ropean Defence Community (EDC), que aunaba política y defensa. Sin embargo, la plasmación práctica de este organismo resultó imposible porque los distintos parlamentos nacionales que hubieran debido aceptar esa nueva estructura no la aprobaron. En Agosto de 1954, la propia Asamblea francesa, país del cual había partido la iniciativa, no ratificó el Tratado que creaba la EDC, lo cual supuso el fin de esta propuesta.

En los primeros meses en que se discutió sobre las ventajas e inconvenientes de esa institución, los laboristas todavía estaban en el poder. Desde el principio se mostraron contrarios a ella, en gran medida porque estaba liderada por los franceses, lo cual hubiera significado cederles la iniciativa en un tema tan vital como la defensa europea, y eso era visto con preocupación desde la perspectiva británica. Por ello ni Attlee ni Bevin apoyaron la EDC. En Marzo de 1851 Bevin tuvo que dimitir por enfermedad y fue sustituido como ministro de exteriores por Herbert Morrison, que se embarcó en una revisión de la causa europea. Durante su mandato el servicio exterior británico adoptó una actitud más favorable a la creación un ejército europeo, sobre todo como fórmula para poder integrar y controlar a la vez el rearme de las fuerzas armadas alemanas ${ }^{39}$. 


\section{Gran Bretaña ante Europa.....}

En esa misma época Attlee escribió un memorandum explicando la política británica respecto a la integración europea en el que se refería también a su actitud ante la integración militar europea: «Nosotros queremos jugar un papel activo en la cooperación europea sobre bases intergubernamentales, pero no estamos dispuestos a limitar nuestra libertad de decisión y de acción a una autoridad supranacional. Animamos a los países que estén dispuestos a adoptar planes en tal sentido, y en el caso del Plan Schuman declaramos nuestro deseo de trabajar estrechamente con él y consideramos la posibilidad de contraer un compromiso mayor en el futuro. Vemos también con simpatía los proyectos para crear un ejército europeo si realmente se demuestra que puede ser efectivo. Igualmente, deseamos desarrollar el papel consultivo del Consejo de Europa, que es el núcleo principal del movimiento de integración europea ${ }^{40}$. Sin embargo, pese al apoyo expreso de los laboristas a una integración militar europea, este partido no llegó a llevarla a la práctica.

Antes de que acabaran las negociaciones en torno a la creación de la EDC los conservadores volvieron al poder en Gran Bretaña. Tal circunstancia se consideró como un hecho positivo para la unidad defensiva europea, dadas las palabras que Churchill acababa de pronunciar ante el Consejo de Europa, en Agosto de 1950, defendiendo la inmediata creación de un ejército europeo bajo un mando unificado, en el cual los británicos debían ser parte importante ${ }^{41}$. No obstante, una cosa eran los discursos desde la oposición, y otra el ejercicio del poder. Cuando volvió a hacerse cargo del Ejecutivo, Churchill se encontró con un partido muy dividido al respecto. Mientras que el ministro de Exteriores, Anthony Eden, declaraba en una asamblea de la OTAN, reunida en Roma en Noviembre de 1951, que Gran Bretaña no participaría en la EDC, el mismo día el ministro del Interior, Sir David Maxwell Fyfe, manifestaba en el Consejo de Europa que el Gobierno de su Majestad era favorable a "tan imaginativo plan» ${ }^{42}$. Lo cierto es que la mayoría del Gabinete y figuras con gran prestigio dentro del partido, como Eden o Salisbury, se mostraban contrarios a la integración militar. También el Foreign Office se opuso a la EDC. De todos los movimientos en favor de una unidad europea, el Plan Pleven fue el que suscitó un mayor rechazo entre la diplomacia británica. La implicación de que en el caso de que Gran Bretaña se incorporara a ese organismo tendría que diluir sus fuerzas armadas en un ejército europeo se consideró una opción inaceptable. Con objeto de evitar esa alternativa, el servicio exterior sugirió que se elaborara una fórmula distinta para organizar una política europea de defensa. 
El primer ministro, Anthony Eden, propuso entonces la creación de la Western European Union (WEU), que agruparía a los seis estados signatarios de la CECA y a Gran Bretaña en un sistema de tratados de defensa mutua, pensados fundamentalmente para prevenir cualquier amenaza que pudiera provenir de Alemania o de la Unión Soviética. Se declaraba que el ejército alemán podría reconstruirse, pero al tiempo se garantizaba que en el caso de que las fuerzas armadas alemanas se volvieran contra otra nación europea, un tratado le aseguraría el apoyo de los demás países signatarios. Además, la WEU respondía a la preocupación británica ante una ofensiva rusa. Para evitar esa posibilidad el Gobierno de Eden consideraba fundamental, primero lograr un pacto de defensa de Europa Occidental, y segundo, conseguir que Estados Unidos lo respaldara. Aunque un objetivo similar ya se había conseguido en parte a través de la firma del Tratado del Atlántico Norte, ratificado el 4 de Abril de 1949, con la WEU se conseguía un eslabón más: que Alemania se incorporara a una alianza militar europea -lo cual reforzaba el potencial defensivo europeo en su frontera oriental frente a Rusia-, y que ello pudiera convertirse en un paso previo a la plena integración alemana en la OTAN. El plan fue aprobado en Octubre de 1954, lo cual fue considerado como una de las pocas victorias que las tesis intergubernamentales defendidas por Gran Bretaña obtuvieron en el proceso de integración europea, presidido en su mayoría por las posturas más proclives al federalismo y al supranacionalismo que sostenían la mayoría de los países continentales ${ }^{43}$.

\section{La entrada en la Comunidad Europea}

A mediados de los cincuenta, Jean Monnet sugirió que el siguiente sector en el proceso de integración debía ser la energía atómica. Gran Bretaña fue invitada a participar en las conversaciones preliminares y de hecho acudió a las reuniones de Messina, iniciadas en Junio de 1955, aunque su participación en las discusiones fue escasa. Gran Bretaña nunca tomó en serio esas conversaciones, ni previó el futuro que se abría tras ellas ${ }^{44}$. De hecho, mientras los demás gobiernos mandaban a representantes tan cualificados como los ministros de exteriores, los británicos enviaron a un funcionario de segunda fila, Russell Bretherton, Senior Official from the Board of Trade. En cualquier caso, la participación británica no duró mucho tiempo. Después de un descanso estival, los países volvieron a reunirse en Bruselas. Y allí informó Bretherton que el Gobierno británico había decidido que no participaría en el mercado común europeo que se estaba discutiendo, por lo cual 
se retiraba de las negociaciones. Las razones aducidas fueron que no deseaban ir más allá de la definición de un área de libre comercio, ni integrarse en una unión aduanera, y sobre todo que se oponían a la naturaleza supranacional de las instituciones previstas. Los resultados de las conversaciones iniciadas en Messina se plasmaron tiempo después en los Tratados de Roma, firmados en Marzo de 1957. Preveían la constitución de la European Economic Energy Community (Euratom) y la European Economic Community (EEC). Aunque ambas instituciones eran esencialmente de carácter económico, los preámbulos de los tratados establecían claramente que el objetivo era la fundación de una unión más estrecha de los pueblos de Europa, en la cual se fundieran economía y política. Con el tiempo la CEE se convertiría en el principal vehículo para la unión europea. El Gobierno de Eden eligió voluntariamente automarginarse del mismo ${ }^{45}$. Gran Bretaña trató de contrarrestar dichas iniciativas creando la European Free Trade Association (EFTA), constituida por los países marginados de la CEE: Suecia, Suiza, Austria, Noruega, Dinamarca, Portugal y la propia Gran Bretaña, que aceptaron coligarse para resolver temas aduaneros sin que ello implicara renunciar a ninguna soberanía nacional.

Probablemente marginarse de la CEE fue el mayor error que Gran Bretaña cometió a la hora de tratar de conseguir el modelo de Europa que prefería: una unión política compuesta por estados soberanos que actuaran conjuntamente con los Estados Unidos; una unión económica en la que se respetara el libre comercio, sin intervencionismo central, y abierta a las transacciones con el resto del mundo; una unión estratégica y defensiva que les proporcionara un peso específico en la escena internacional. En vez de luchar por alcanzar ese modelo negociando las condiciones con las demás potencias europeas desde dentro de una estructura común, el Gobierno británico optó por torpedear las conversaciones de Messina, apostando en su lugar por la OECE como foro de negociación y cooperación. Esta política se reveló como una importante falta de juicio político que condujo a la exclusión de Gran Bretaña del proceso europeo que entonces comenzaba ${ }^{46}$.

Sólo cuatro años después de la firma de los Tratados de Roma, Gran Bretaña reconoció su error y se mostró dispuesta a integrarse en Europa. En Julio de 1961, Harold MacMillan presentó la primera solicitud para conseguir tal objetivo ${ }^{47}$. A ello le inclinaban numerosas razones: primero, el primer ministro Eden, hostil a cualquier forma de participación británica en una unión europea, fue reemplazado por MacMillan, un hombre pragmático y más favorable a la integración. Segundo, la economía británica estaba pasando por una época de re- 


\section{$M^{a}$ Dolores Elizalde}

cesión, y por contra la CEE estaba tiendo un notable éxito económico. Pocos años después de su fundación, los Estados miembros habían acordado unas tarifas aduaneras exteriores, y tenían una política agraria e industrial común que favorecía el desarrollo del conjunto. Tercero, distintas crisis internacionales, como la de Suez de 1956 o la de Africa del Sur de 1961, habían evidenciado la creciente soledad británica en el mundo internacional. El apoyo de la Commonwealth no tenía peso suficiente cuando en ese contexto se producía una situación crítica. A ello se unía que los lazos con Estados Unidos se iban distendiendo, en un proceso paralelo al acercamiento norteamericano a la Comunidad Económica Europea, contemplado con aprehensión por los británicos. Si Gran Bretaña quería aumentar su influencia internacional, debía integrarse en la CEE y desde esa institución convertirse en el socio principal de los norteamericanos ${ }^{48}$.

Desde mediados de los años cincuenta se fue haciendo cada vez más evidente que la posición internacional de Gran Bretaña había variado sustancialmente. Había perdido definitivamente la hegemonía que un día tuvo en ese campo y tenía que acoplarse a un nuevo contexto definido por la existencia de dos superpotencias enfrentadas que se habían repartido el mundo en aréas de influencia ajenas a la impronta británica. Había perdido también su Imperio y los fuertes lazos políticos y económicos que la unían a territorios repartidos por todos los océanos. La Commonwealth le proporcionaba todavía un foro y una repercusión internacional destacada, pero lejos ya del papel preponderante que había desempeñado en la escena mundial. Esas circunstancias provocaron que aumentaran los deseos británicos de integrarse en un conjunto poderoso, y ninguno mejor que el europeo ${ }^{49}$. Sin embargo, la integración británica se vió dificultada por sus explícitas pretensiones de convertirse en uno de los líderes del proceso europeo, circunstancia contemplada con enorme recelo por los países que ya formaban parte del núcleo inicial de la comunidad europea, y muy especialmente por Francia ${ }^{50}$. Estuvo obstaculizada también por el entendimiento establecido casi desde el primer momento entre Francia y Alemania. Gran Bretaña quiso convertirse en el tercer vértice de un triunvirato dirigente, pero no consiguió alcanzar ese objetivo. Nunca llegó a producirse una especial relación a tres bandas entre Francia, Alemania y Gran Bretaña, y ésta última se sintió marginada de la cúpula europea ${ }^{51}$.

La Francia de Charles de Gaulle había alcanzado un papel predominante en el seno de la Comunidad y lo último que deseaba era la participación británica en esta institución. Con Gran Bretaña en 
la CEE podría haber un líder rival que se opusiera a las directrices francesas. Por ello, en 1963, De Gaulle vetó la entrada británica en la Comunidad alegando que Gran Bretaña no estaba preparada para una «vocación europea» y que actuaría como un caballo de Troya americano dentro de esta organización.

En Mayo de 1967 el nuevo premier británico laborista, Harold Wilson, presentó la segunda solicitud británica para entrar en la CEE. Las consideraciones que le llevaron a tal decisión fueron la crisis económica en que Gran Bretaña estaba inmersa; la tendencia de los países miembros de la Commonwealth a desarrollar sus propias relaciones comerciales con otras potencias distintas de Inglaterra; las dificultades de la política aduanera, en la cual Gran Bretaña se veía obligada a aceptar las altas tarifas exteriores de la CEE, mientras que no se podía beneficiar de las reducidas medidas interiores de ese grupo. Junto a estas razones económicas, persistía el deseo británico de redefinir su posición internacional contando con el apoyo del grupo europeo. Su influencia en el mundo era cada vez menor y su posición ya no le proporcionaba las ventajas que antes le había procurado el libre comercio. Además, el obligado relevo de Kennedy por Johnson en la Presidencia de los Estados Unidos había producido un nuevo alejamiento de la Administración norteamericana, más volcada entonces en asuntos internos, y Gran Bretaña volvía a sentir el peso de la soledad. Sin embargo, se repitió la situación anterior y en Diciembre del $67 \mathrm{De}$ Gaulle volvió a vetar la integración británica aduciendo su excesiva cercanía a los Estados Unidos. Sólo después de la salida del gobierno del dirigente francés, en Mayo de 1969, se abrió el camino a la integración británica en la CEE.

Después de la dimisión de De Gaulle el proceso fue rápido. En el Consejo de Ministros de la CEE de Julio de 1969 se planteó el tema de ampliar los miembros de la Comunidad y Francia informó que no presentaría un nuevo veto. Gran Bretaña indicó que estaba preparada para abrir las negociaciones en cuanto los Seis dieran el visto bueno para ello. Las conversaciones para ello se iniciaron el 30 de Junio de 1970 en Luxemburgo. El principal problema era la negociación respecto a las contribuciones británicas al presupuesto comunitario -a los británicos les parecía inaceptable la reciente decisión adoptada en La Haya para crear un presupuesto europeo común, pero prefirieron ignorar el asunto en ese momento en aras del objetivo final: una integración que a todas luces se había postergado excesivamente-. La estrategia seguida para ello por Edward Heath fue incorporarse a la Comunidad tan pronto como fuera posible, soslayar los problemas potenciales, y 


\section{$M^{a}$ Dolores Elizalde}

negociar posteriormente cualquier diferencia, ya desde el seno de esta institución. Finalmente, tras dieciocho meses de negociación, se firmó en Bruselas un acuerdo para la entrada británica, y el 1 de Enero de 1973 Gran Bretaña se convirtió en miembro de la Comunidad Europea. Sin embargo la integración no se reveló nada fácil. De 1974 a 1984 el proceso de adaptación de Gran Bretaña a la CEE dominó los asuntos comunitarios ${ }^{52}$. Los términos de su integración tuvieron que ser renegociados y se discutieron hasta la saciedad los ajustes económicos y presupuestarios que requería la participación británica, hasta llegar a acuerdos satisfactorios para todas las partes. También es justo señalar que los problemas económicos en la Comunidad no se derivaron sólo de la integración británica, sino de la seria crisis económica que todos los países tuvieron que afrontar en ese período. A partir de mediados de los ochenta las relaciones fueron más fáciles y la integración empezó a ser fructífera. Hoy en día, con el Gobierno de Tony Blair Gran Bretaña parece incluso haberse convertido en uno de los líderes de la política de la Unión Europea, reemplazando el antiguo predominio franco alemán gracias al apoyo de países como España, Italia y Portugal.

\section{Notas}

1 Circunstancia que sí influía en países más inestables, menos desarrollados o con una historia reciente más turbulenta, caso de España, Portugal o Grecia. En palabras de Simon Bulmer: «The absence of any equivalent political motivation for British involment in European integration is of great importance in explaining British reluctance to participate in supranational integration", "Britain and European Integration: Of Sovereignity, Slow Adaptation and Semi-Detachment», en Stephen George, ed., Britain and the European Community, Oxford, Clarendon Press, 1992, p. 17. C. Tugendhat también niega la existencia de este sentimiento en la conciencia británica: Making Sense of Europe, Harmondsworth, 1987.

2 Simon Bulmer, Stephen George y Andew Scott, eds., The United Kingdom and EC Membership Evaluated, London, Pinter Publishers, 1992. En esta obra se defiende la tesis de que Gran Bretaña siempre quiso ser el nervio central de un Imperio mundial, o al menos de una comunidad de naciones basadas en la libre cooperación, que era lo que representaba la Commonwealth después de la Segunda Guerra Mundial. A los británicos les parecía mucho más atractivo convertir a la Commonwealth en el elemento principal de su política mundial, y que ese fuera su foro fundamental de actuación exterior, que no la idea de convertirse en un engranaje más de una comunidad europea.

3 Cristopher Hill, «The Historical Background: Past and Present in British Foreign Policy", en M. Smith, S. Smith y B. White, eds., British Foreign Policy: Tradition, Change and Transformation, London, 1988. W. Hanrieder y G. Auton, The Foreign Policies of West Germany, France and Britain, New Jersey, Englewood Cliffs, 1980. 


\section{Gran Bretaña ante Europa.....}

«It should not be forgotten, first of all, that the traditional pattern of British European policy had been to attempt to maintain domestic security through playing the continental powers off against each other»...«The early recognition of the inability to continue as guarantors of stability in the Eastern Mediterranean, resulting in American commitments through the Truman Doctrine, was one example. Another was the realization that the former British strategy of playing continental powers off against one another was no longer appropriate in a bipolar world", Simon Bulmer, "Britain and European Integration: Of Sovereignity, slow Adaptation and Semi-Detachment», en Stephen George, ed., Britain and the European Community, Oxford, Clarendon Press, 1992, pp. 14 y 5 .

4 De alguna manera los años de la seguridad colectiva habían supuesto un precedente para la articulación de una Europa unida. Desde el punto de vista británico, la búsqueda de una seguridad colectiva en la década de los veinte había tenido tres propósitos. Uno era tratar de contener un posible revisionismo alemán y mantener las fronteras establecidas en el tratado de Versalles; lo cual podía contemplarse como un elemento defensivo y era de particular concernimiento de los franceses. El segundo era rehabilitar a Alemania y posibilitar que se reincorporara a la diplomacia europea. El tercero era tratar de resolver las rivalidades a través de organismos internacionales. El multilateralismo debía remplazar al bilateralismo y sobre todo al unilateralismo. Ejemplos prácticos de esa política fueron los Acuerdos de Locarno, firmados en 1925 por Gran Bretaña, Francia, Alemania, Bélgica e Italia; el pacto de Kellogg-Briand, ratificado en 1928 por 65 Estados que condenaron el recurso a la guerra como forma de resolver las rivalidades internacionales y renunciaron expresamente a ella como un instrumento de política nacional en sus relaciones con las demás potencias; o la Sociedad de Naciones, que significaría la culminación de esa orientación. Para Gran Bretaña la política de seguridad colectiva fue positiva porque revivió el internacionalismo como fórmula para resolver los conflictos, lo cual le permitía no tener que asumir la última responsabilidad de la paz colectiva en Europa. Por ello, en cierta medida, puede considerarse como un precedente de la Unión Europea en tanto que significaban organismos intergubernamentales que regulaban los problemas planteados, sólo que en el caso de la Comunidad los planteamientos se ciñeron a Europa Occidental y se extendieron a cuestiones políticas, económicas y de cooperación, desbordando el ámbito militar e internacional.

5 Stephen George, Britain and European Integration since 1945, Oxford, Blackwell, 1991, p. 33.

6 Avi Shlaim, Peter Jones y Keith Sainsbury, British Foreign Secretaries since 1945, Newton Abbot, 1977.

7 John Young, Britain, France and the Unity of Europe, Leicester, Leicester University Press, 1984

8 «Of the larger member states, Britain is unique in having a continuing aversion or scepticism towars integration»..."An important distinction between co-operation and supranational integration, i.e. where sovereignty is transferred to a level above the nation state. Britain was prepared, in the 1940 s and $1950 \mathrm{~s}$, to contemplate cooperation but not the transfer of sovereignty associated with the projects for integration"... «Hence, just as there has been an aversion in British élite and public opinion to ceding sovereignty, so there has been an aversion to perceiving integration as a political process. This is a major contrast with the other large EC member states, which make great paly of the grand ideals of European Union and still manage successfully to embody 
national interests in their vision! For Britain, by contrast, national interests and political integration are regarded as diametric opposites" Simon Bulmer, "Britain and European Integration: Of Sovereignity, slow Adaptation and Semi-Detachment», en Stephen George, ed., Britain and the European Community, Oxford, Clarendon Press, 1992, pp. 3, 5 y 11 .

9 "Why has this question of sovereignty been of such importance to British policy? There are numerous historical explanations: the continuity of institutions since the English Civil War; former world-power satus; the successful avoidance, as an island, of fullscale invasion; the position of having "stood alone" in 1940 together with the prestige gained as a victor; the myth of parliamentary sovereignty; pride in national identity as an adversion to "homogenization" by European social integration (arguably a variant of public concerns about inmigration); and perhaps even popular loyalty to the Crown", Simon Bulmer, Op. cit., p. 9. También acerca de este tema: Willian Wallace: "What Price Independence? Sovereignty and Interdependence in British Politics», International Affairs, 62, (1986).

10 «La Europa que esa gente ha creado se ha convertido en un leviathan antiliberal y burocrático, obsesionado por la armonización", juicio de Ralf Dahrendorf citado por un parlamentario conservador, Parliamentary Debates, 21 Octubre 1981, vol. 823, col. 942.

11 Ejemplificando ese temor, en Enero de 1972, un parlamentario laborista llevó a la Cámara 42 volúmenes conteniendo 2.500 regulaciones de la Comunidad que se convertirían automáticamente en leyes inglesas en el momento en que Gran Bretaña firmara el Tratado de Roma, Parliamentary Debates, 20 Enero 1972, vol. 829, cols. 678-80.

12 "The importance (of ECC) for the UK has been based on pragmatism rather than principle...For successive British Governments, objectives consisted in achieving European Security and outlets for trade; both proved to be possible in the short term withou supranational integration. To have adopted an idealistic political commitment to integration would have been a negative step: a questioning of Britiain's independent satus in international politics», Simon Bulmer, Op. cit., p. 17.

13 Alan Butt Philip, "British Preassure Groups and the European Community", y Neill Nugent, «British Public Opinion and the European Community, en S. George, ed., Britain and the European Community. The politics of Semi-Detachment, Oxford, Clarendon Press, 1992, pp. 149-171 y 172-201 respectivamente. Hansard Society, The British People: Their Voice in Europe, Farnborough, 1977.

14 En este sentido, desde una perspectiva española, cabe señalar el fuerte contraste con el estado de opinión existente en España, para la cual Europa fue hasta 1986 -en que logró la integración en la Comunidad- el modelo a imitar, la meta que alcanzar. No hay más que recordar la literatura regeneracionista y la gráfica expresión de Ortega «España como problema, Europa como solución». Desde comienzos de siglo y hasta los años más recientes, asentada ya en España la democracia, esa línea de pensamiento ha estado muy presente en los discursos de los políticos e intelectuales españoles.

15 Cuando MacMillan llegó a la conclusión de que Gran Bretaña debía solicitar la incorporación a la Comunidad, presentó su decisión al país y al Parlamento en términos estrictamente prácticos. No intentó reemplazar el fuerte sentimiento de identidad nacional que todavía existía en Gran Bretaña por un alternativo sentido del deber europeísta. La opinión pública británica difícilmente hubiera aceptado ese tono.

16 Nigel Ashford, "The Political Parties», en S. George, ed., Britain and the European Community. The politics of Semi-Detachment, Oxford, Clarendon Press, 1992, 


\section{Gran Bretaña ante Europa.....}

pp. 119-148. Stephen George, Britain and European Integration since 1945, Oxford, Blackwell, 1991, pp. 67-73, T. Lindsay y M. Harrington, The Conservative Party, 19181879, London, 1979. Z. Layton-Henry, Conservative Party Politics, London, 1980.

17 Stephen George, Britain and European Integration since 1945, Oxford, Blackwell, 1991, pp. 73-83. J. Grahl y P. Teague, «The British Labour Party and the European Community», Political Quarterly, 59, (1988), 72-85. L. Robins, The Reluctant Party: Labour and the EEC, 1961-1975, Ormskirk, 1979.

18 R.J. Harrison, Europe in Question: Theories of regional International Integration, London, Allen\&Unwin, 1974, p. 47. Walter Liepgens, A History of European Integration, vol 1, 1945-1947: The Formation of the European Unity Movement, Oxford, Clarendon Press, 1982, pp. 44-58.

19 Esta actitud levantó críticas entre los propios británicos. Anthony Nutting, el anterior ministro de exteriores conservador, apuntó en sus memorias, significativamente llamadas Europa no esperará, que después de la Segunda Guerra Mundial Gran Bretaña podía haberse hecho con el liderazgo de la Europa occidental sin problemas, pero que en aquel entonces el país estaba gobernado por hombres -los laboristas de Attleecuya filosofía política era muy limitada y que no pudieron ser más insulares ni más nacionalistas: prefirieron el insularismo al internacionalismo. A. Nutting, Europe will not wait: a warning and a way out, London, 1960, pp. 3-5. No parece que tales palabras respondan totalmente a la verdad, según los hechos que veremos a continuación, pero son ilustrativas de un estado de opinión.

20 V. Rothwell, Britain and the Cold War, 1941-1947, London, 1982, p. 414.

21 Public Record Office (PRO), FO 371- 50826, U1768, 19 Marzo 1945, cit. por Geoffrey Warner, "The Labour Goverments and the Unity of Western Europe", en Ritchie Ovendale, ed., The Foreign Policy of the British Labour Governments, 1945-1951, Leicester University Press, 1984, p. 62.

22 PRO, FO 371- 67724, Z4670, carta de Sargent, 16 Mayo 1947, cit. por Geoffrey Warner, p. 63.

23 PRO, FO 371- 67724, Z5705/G, 4 Junio 1947, cit. por Geoffrey Warner, p. 64.

24 «This could only be prevented if we are prepared with measures which, involving economic ties, would bind Western European countries politically more closely to ourselves...We must also consider the position in relation to Western Europe as a whole", PRO, CAB 129/16, cit. Warner, p. 64.

25 «It seems to me a very unrealistic proposal. The Colonies probably could not afford it; The Dominions, who wish to build up their own industries, do not want it; and the Americans, who would be left out the cold, would surely resent it. It would dislocate domestic production in all the countries concerned. It would completely abolish Imperial preference», escribía Lintott, un funcionario del Tesoro, PRO, CAB 129/16, carta del 23 Mayo 1947, cit. Warner, p. 65. A la vista de tan negativas consideraciones, se encargó que un grupo de economistas de Cambridge elaborararan un informe sobre la conveniencia o perjuicio de una unión económica europea. Pero antes de que pudieran presentar sus conclusiones se conoció el Plan Marshall que marcó inmediata línea de actuación en temas económicos europeos.

26 El 22 de Septiembre de 1947 tuvo lugar una reunión entre Bevin y el primer ministro francés, M. Ramadier, en la cual el premier británico defendió que si Europa occidental se unía podría ser tan poderosa como la Unión Soviética o los Estados Unidos: "Only owing to their divisions that the Western democracies did not occupy the position that they might in the world today. In addition to their populations they 
possessed between them supplies of raw materials grater than those of any other country". PRO, FO 371, memorandum de la conversación entre Bevin y Ramadier, 22 Septiembre 1947; minuta de Hogg para el Foreign Office, 27 Septiembre 1947; minuta de Wilson, 8 Octubre 1947, cit. Warner, p. 66.

27 El 2 de Marzo de 1948 Bevin explicó a sus colaboradores: «We ought to aim a joint multi-lateral economic, social and defensive treaty without any previous guarantee of American backing», PRO, minuta de Jebb, 3 Marzo de 1948, cit. Warren, p. 67.

28 "The British Ambassador in Paris was informed on 28 August that Bevin felt 'that on this issue the French Government are playing politics, that they have not really given any serious study to the matter and that he declines to be bustled into folly, simply to suit the temporary convenience of the French Government», PRO, FO 371, carta de Kirkpatrick del 28 Agosto de 1948, cit. Warren, p. 68.

29 "Only by such acceptance was any progress ever likely to be made in the direction of a closer unity which would include Great Britain", cit. Warren, p. 69.

30 «General debates, followed by no action, and fruitless controversies about federal constitutions, would make the peoples lose heart. What we needed now was solidarity without a constitution. Decisions must be kept in the hands of governments and their representatives... There was a danger of a crash between the parliamentary assembly and governments. But since governments would be represented on the Council of Europe, there would be no such danger of a crash between the governments and the Council", Dalton Diary, 19 November 1948, cit. Warren, p. 70.

31 "Thus, América and the Commonwealth were judged to be more vital than Europe to British economic considerations", John Young, Britain, France and the Unity of Europe, Leicester, Leicester University Press, 1984, pp. 37-41.

32 Avi Shlaim, Peter Jones y Keith Sainsbury, British Foreign Secretaries since 1945, Newton Abbot, 1977, p. 42.

33 PRO, CAB 134, Documento del Economic Policy Committee of the Cabinet sobre la política británica hacia la OEEC, elaborado conjuntamente por Bevin y Sir Stafford Cripps, Chancellor of the Exchequer, 26 Enero 1949.

34 PRO, CAB 128, Nuevo documento conjunto de Bevin y Cripps, 25 Octubre 1949.

35 En una reunión mantenida el 10 de Noviembre de 1949 entre los responsables de exteriores de Gran Bretaña, Francia y Estados Unidos, Bevin, Schuman y Acheson, el primero de ellos declaró que Gran Bretaña nunca sería enteramente un país europeo y que no deseaba verse en la tesitura de tener que elegir entre su relación con la Commonwealth y con Europa occidental porque el resultado sería perjudicial para todos ellos. La respuesta de Schuman fue especialmente generosa, pues señaló que Europa era inconcebible sin Gran Bretaña y que por tanto los británicos no debían sentir que se les hacía elegir entre una u otra formación, sino que todos los movimientos que se realizaran en favor de la unión europea debían permitir a los ingleses una síntesis entre ambas opciones.

36 "The Schuman Plan was rejected by Britain because of its supranational challenge to sovereignty", Simon Bulmer, "Britain and European Integration: Of Sovereignity, slow Adaptation and Semi-Detachment», en Stephen George, ed., Britain and the European Community, Oxford, Clarendon Press, 1992, p. 6. Hasta tal punto se sentía rechazo hacia esos planteamientos que cuando se discutió la conveniencia de apoyar el Plan Schuman el Labour's National Executive declaró que se negaban a convertirse en "una pequeña y poblada isla de la costa Oeste de la Europa continental».

37 Kenneth Morgan, Labour in Power, 1945-1951, Oxford, Clarendon Press, 1984, p. 419. George, op. cit. p. 38. Barker defiende en The British Between the Superpowers, 


\section{Gran Bretaña ante Europa.....}

1945-1970, London, MacMillan, 1983, que la no participación de Gran Bretaña en el Plan Schuman puede considerarse como un turning point en las relaciones británicas de postguerra con la Europa occidental. Era el primer éxito de una iniciativa británica tomada sin Gran Bretaña, y de alguna manera, contra Gran Bretaña, p. 203. Esta misma autora también trata este tema en Britain in a Divided Europe, 1945-1970, London, Weidenfeld\&Nicolson, 1971.

38 «No British Government could be expected to accept such a commitment without having had any opportunity to assess the consequences which it might involve for our key industries, our export trade and our level of employment». PRO, CAB 128, 2 June 1950, cit. Warren, p. 72. "It's no good, we cannot do it, the Durham miners won't wear it", exclamó Herbert Morrison, Lord President of the Council y número dos del Gobierno, al conocer el ultimatum francés. Incluso Kenneth Younger, segundo de a bordo del Foreign Office y el único que argumentó en favor de aceptar los requerimientos franceses, apuntó aquél día en su diario «On reflection, I really think we had no choice".

39 «The European Army concept, as advocated by the French and now strongly supported by the Americans, is primarily a method of insuring that any German defence contribution shall be inextricably bound up with Western European defence as a whole and prevented from leading to the creation of German armed forces", Attlee en la reunión del gabinete del 30 de Agosto de 1951, cit. Warren, p. 77.

40 PRO, CP (51), 30 Agosto 1951, cit. Warren, p. 78.

41 Anthony Eden, Memoirs. Full Circle, London, Cassell, 1960, p. 30.

42 Anthony Sampson, Macmillan. A Study in Ambiguity, London, Allen Ale, 1967, p. 90.

43 Posteriormente, cuando en 1966 De Gaulle decidió que Francia abandonara la OTAN, la-WEU se convirtió también en un forum que permitía la elaboración de una estrategia común entre los países europeos que eran miembros de la OTAN y una Francia independiente. De alguna manera, abrió el camino a una respuesta militar europea, ajena a Estados Unidos.

$44 \mathrm{Y}$ ello a pesar de voces como la de Harold Macmillan, Chancellor of the Exchequer por aquel entonces, que en carta a Sir Edward Bridges, Senior Civil Servant at the Treasury, manifestaba que quizás en Messina no se llegara a nada, pero que temía un mundo dividido en la esfera rusa, la esfera americana y una Europa unida de la cual Gran Bretaña no fuera miembro, Harold Macmillan, Riding the Storm, 1956-1959, London, Macmillan, 1971, p. 74. También el Gobierno norteamericano señaló al británico la importancia que podrían adquirir los acuerdos de Messina. Pero Eden, que había sucedido a Churchill como primer ministro, consideró que no tenían en cuenta los perjuicios que le causaría a Gran Bretaña participar en el proyecto que se estaba elaborando ni el riesgo de que se adoptaran unas tarifas aduaneras perniciosas para la economía británica que en el caso de integrarse no tendría otro remedio que aceptar. Además creyó que los norteamericanos con sus recomendaciones trataban de apartar a los ingleses de la Commonwealth, sabiendo que su pertenencia al grupo europeo dificultaría su identificación con aquella otra organización.

45 Neill Nugent, The Government and Politics of the European Community, London, Macmillan, 1989.

46 Harold Macmillan explicaba en sus Memorias que el Gobierno no creyó que esa fuera la única vía posible hacia la integración europea: «Cuando fui elegido Secretario de Asuntos Exteriores, en la primavera de 1955, no parecía que hubiera un único camino hacia la unidad europea, sino distintas vías, a veces paralelas, a veces en- 
trecruzadas ¿Iba a extenderse la cooperación a la Alianza del Atlántico Norte? ¿Iba a reemplazar esa posibilidad a un ideal estrictamente europeo? ¿Iba Europa, ya dividida entre Este y Oeste, comunista y libre, a ser dividida de nuevo entre las tres grandes potencias continentales -Francia, Alemania e Italia-, y junto a ellas Holanda, Bélgica y Luxemburgo, por un lado, y Gran Bretaña liderando al resto de la Europa occidental, por otro? ¿descansaría el futuro de Europa en la OEEC, más comprehensiva con sus miembros que el propio Consejo de Europa, o se basaría la unidad en el Plan Schuman? Macmillan, op. cit., pp. 64-65.

47 Richard Bailey, The European Connection, Oxford, Pergamon, 1983. Miriam Camps, Britain and the European Community: 1955-1963, Oxford, Oxford University Press, 1964. F. Northedge, Britain and the ECC: Past and Present, en R. Jenkins, ed., Britain and the EEC, London, 1983. S. George, An Awkward Partner: Britain in the European Community, Oxford, 1990. S. George, "The Policy of British Governments within the European Community", en S. George, ed., Britain and the European Community. The politics of Semi-Detachment, Oxford, Clarendon Press, 1992, pp. 30-63. S. Bulmer, «Domestic politics and European Community Policy-Making», Journal of Common Market Studies, 21, (1983), 349-363.

48 En 1961 la nueva Administración Kennedy trató de animar a Gran Bretaña para que entrara en la $\mathrm{CEE}$, con objeto de que contrarrestara en esa institución las directrices marcadas por De Gaulle, cada vez más incisivo en una política europea fuerte e independiente, lo cual hizo crecer en Estados Unidos el temor a la consolidación de una tercera fuerza mundial neutralista que pudiera distorsionar el orden establecido. Kennedy señaló a MacMillan que desde dentro de la CEE Gran Bretaña podría maniobrar mejor para conseguir la política aduanera y comercial que más les conviniera y además podría colaborar con Estados Unidos para lograr el orden internacional más favorable para todos. Arthur Schlesinger, A Thousand Days: John F. Kennedy in the White House, London, Andre Deutsch, 1965, p. 720.

49 El White Paper de Julio de 1971, que evaluaba las ventajas de una posible incorporación a la $\mathrm{CE}$, señalaba: «En una generación hemos tenido que renunciar a nuestro pasado imperial y nos hemos visto rechazados de un futuro europeo. Nos hemos quedado sin aliados, los cuales contemplan con la misma incertidumbre que nosotros nuestro futuro papel y lugar en el mundo", The United Kingdom and the European Communities, HMSO, Cmnd., 4715, July 1971, p. 17, cit. en Simon Bulmer, Stephen George y Andew Scott, eds., The United Kingdom and EC Membership Evaluated, London, Printer Publishers, 1992, pp. 4-5. Estos autores, compartiendo una opinión muy extendida, señalan que la principal razón para la incorporación británica a la Comunidad fue ante todo el temor a la exclusión, a quedar fuera de un foro prometedor. En el largo debate que se mantuvo en el Parlamento británico en el otoño de 1971, en el cual se debatía sobre el sentido de la integración británica, Alec Douglas-Home y Edward Heath señalaron la importancia que tenía, dentro de un contexto internacional marcado por dos grandes superpotencias, estar dentro de un bloque continental poderoso. El argumento principal fue que el mundo estaba cambiando y que Gran Bretaña debía incorporarase a algún grupo con peso específico si no quería quedarse aislada y sin voz. De otra manera, el futuro sería decidido por otros y los británicos no podrían tener ningún control sobre el proceso.

50 Esa confianza en convertirse en líderes del proyecto europeo cuando se produjera su integración se reflejó en la observación que el ministro de Asuntos Exteriores británico, George Brown, le hizo a Willy Brandt: «Willy, debes meternos, para que 


\section{Gran Bretaña ante Europa.....}

así nosotros podamos tomar el mando", Willy Brandt, People and Politics. The Years 1960-1975, London, 1978, p. 161.

51 Desde fines de los cuarenta De Gaulle abogó por una Europa occidental fuerte e independiente económica y políticamente de Estados Unidos. Para poder desarrollar esa línea de acción el presidente francés buscó el apoyo alemán y firmó un acuerdo de amistad con Adenauer, que evidenciaba el propósito francés de ganarse a los alemanes como aliados para construir la Europa en la que Francia creía. Desde entonces, -y hasta fechas muy recientes en que el diálogo entre Chirac y Shröeder parece haberse distendido-, el eje franco-alemán ha sido uno de los pilares de la construcción europea, W. Wallace, ed., Britain in Europe, London, 1980.

52 Stephen George, Britain and European Integration since 1945, Oxford, Blackwell, 1991, p. 24. 